\title{
Meson Structure in a Relativistic Many-Body Approach
}

\author{
Felipe J. Llanes-Estrada and Stephen R. Cotanch \\ Department of Physics, North Carolina State University, Raleigh, North Carolina 27695-8202
}

(Received 11 June 1999)

\begin{abstract}
Results from an extensive relativistic many-body analysis utilizing a realistic effective QCD Hamiltonian are presented for the meson spectrum. A comparative numerical study of the BCS, Tamm-Dancoff (TDA), and RPA treatments provides new, significant insight into the condensate structure of the vacuum, the chiral symmetry governance of the pion, and the meson spin, orbital, and flavor mass splitting contributions. In contrast to a previous glueball application, substantial quantitative differences are computed between TDA and RPA for the light quark sector with the pion emerging as a Goldstone boson only in the RPA.
\end{abstract}

PACS numbers: 12.39.Pn, 11.10.St, 12.39.Ki, 12.39.Mk

Common to the diverse areas of condensed matter, molecular, atomic, and nuclear physics is the routine implementation of many-body techniques such as the Bardeen, Cooper, Schrieffer (BCS), Tamm-Dancoff (TDA), and random phase approximation (RPA) methods. Particle physics, with an inherent few-body nature, has generally been devoid of such applications even though hadronic structure, requiring a relativistic QCD description, is an extremely challenging many-body problem. The purpose of the present Letter is to report a comparative study documenting the powerful utility of the above techniques for hadronic systems and to detail new, important meson structure results clarifying the nature of spin splittings and the role of chiral symmetry. The equations of motion, while numerically solvable, exhibit a richness and complexity beyond the simple two-body equations such as the generalized Schrödinger schemes. We find that both TDA and RPA solutions to an approximate QCD Hamiltonian with linear confinement reproduce the meson spectrum except for the pion, where only the RPA reasonably describes the mass and decay constant due to proper implementation of chiral symmetry.

This work complements our previous many-body treatment [1] of the gluonic sector in which the lattice gauge "measurements" were reproduced. Our collaborative program seeks to develop a rigorous effective Hamiltonian from QCD and then to comprehensively investigate hadronic structure by systematic, accurate diagonalization utilizing controllable approximations. Reference [2] details our renormalization program, based upon a continuous cutoff regularization and similarity transformation. That work addressed only the gluon sector but a similar effort is currently in progress for the quark sector. Accordingly, this paper presents many-body solutions for only the unrenormalized effective Hamiltonian. The starting point is the approximate QCD quark Hamiltonian in the Coulomb gauge

$$
\begin{aligned}
H= & \int d \vec{x} \Psi_{q}^{\dagger}(\vec{x})\left(-i \vec{\alpha} \cdot \vec{\nabla}+\beta m_{q}\right) \Psi_{q}(\vec{x}) \\
& -\frac{1}{2} \int d \vec{x} d \vec{y} \rho^{a}(\vec{x}) V(|\vec{x}-\vec{y}|) \rho^{a}(\vec{y}),
\end{aligned}
$$

involving the quark field $\Psi_{q}(\vec{x})$, current quark mass $m_{q}$, and color density $\rho^{a}(\vec{x})=\Psi_{q}^{\dagger}(\vec{x}) T^{a} \Psi_{q}(\vec{x})$. Coupling to the gluonic sector is omitted and the Faddeev-Popov determinant is replaced by its lowest order unit value. Consistent with our previous work [1], the confining potential is a linear interaction, $V=\sigma|\vec{x}-\vec{y}|$, rather than the harmonic oscillator $[3,4]$ since lattice gauge theory generates this form with slope (string tension) $\sigma=0.18 \mathrm{GeV}^{2}$. Instead of a simplified gap differential equation for the harmonic oscillator potential, we solve a numerically quite sensitive nonlinear integral equation (see below) and reproduce earlier results [5]. The density-density two-body form permits only color singlets in the physical spectrum as other $\mathrm{SU}_{c}(3)$ representations are shifted to infinite energy.

Next we introduce our first many-body improvement by performing a BCS rotation (similarity transformation) from the bare (undressed) quark basis to an improved quasiparticle basis. This entails rotated spinors in the quark

$$
\begin{aligned}
U_{\lambda}(\vec{k}) & =\frac{1}{\sqrt{2}}\left[\begin{array}{c}
\sqrt{1+\sin \phi(k)} \chi_{\lambda} \\
\sqrt{1-\sin \phi(k)} \vec{\sigma} \cdot \hat{k} \chi_{\lambda}
\end{array}\right], \\
V_{\lambda}(\vec{k}) & =\frac{1}{\sqrt{2}}\left[\begin{array}{c}
-\sqrt{1-\sin \phi(k)} \vec{\sigma} \\
\sqrt{1+\sin \phi(k)} \chi_{\lambda} \chi_{\lambda}
\end{array}\right],
\end{aligned}
$$

field expansion with quasiparticle operators $B, D$ instead of bare operators $b, d$ (see Ref. [4] for details)

$$
\Psi_{q}=\sum_{\lambda} \int \frac{d \vec{k}}{(2 \pi)^{3}}\left[U_{\lambda}(\vec{k}) B_{\lambda}(\vec{k})+V_{\lambda}(-\vec{k}) D_{\lambda}^{\dagger}(-\vec{k})\right] e^{i \vec{k} \cdot \vec{x}}
$$

The spin state is denoted by $\lambda$ and color indices are suppressed. The gap angle $\phi(k)$ governs the BCS vacuum, $|\Omega\rangle$, defined by $B_{\lambda}|\Omega\rangle=D_{\lambda}|\Omega\rangle=0$. This vacuum, a coherent state containing quark condensates (Cooper pairs), is an improvement over the trivial vacuum. The gap angle is obtained variationally by minimizing the vacuum (ground state) energy $\delta\langle\Omega|H-E| \Omega\rangle=0$ yielding the gap equation which is similar to the Schwinger-Dyson 
equation for the quark self-energy in the rainbow approximation

$$
\begin{aligned}
k s_{k}-m_{q} c_{k}= & \frac{2}{3} \int \frac{d \vec{p}}{(2 \pi)^{3}} \hat{V}(|\vec{k}-\vec{p}|) \\
& \times\left[s_{k} c_{p} \hat{k} \cdot \hat{p}-s_{p} c_{k}\right],
\end{aligned}
$$

where $\hat{V}(|\vec{k}-\vec{p}|)=-8 \pi \sigma|\vec{k}-\vec{p}|^{4}$ is the linear potential in momentum space. The solution $s_{k}=\sin \phi(k), c_{k}=$ $\cos \phi(k)$ also provides the quark condensate $\langle\bar{q} q\rangle$

$$
\langle\bar{q} q\rangle=\langle\Omega|\bar{\Psi}(0) \Psi(0)| \Omega\rangle=-\frac{3}{\pi^{2}} \int d p p^{2} s_{p},
$$

in the BCS vacuum. From the gap equation at large $k$, $s_{k} \rightarrow m_{q} / \sqrt{m_{q}^{2}+k^{2}}$, yielding a quadratically divergent condensate for nonzero current quark mass which must be renormalized [6]. For $m_{q}=0$ we compute $\langle\bar{q} q\rangle \simeq$ $-(113 \mathrm{MeV})^{3}$. We also added the Coulomb $\frac{1}{r}$ interaction corresponding to one gluon exchange, with a reasonable cutoff, and found a slight improvement to 119 , in agreement with the more elaborate, renormalized result of [6] but still substantially less than lattice theory results $(\approx 250)$. The BCS vacuum also exhibits spontaneous chiral symmetry breaking resulting in a constituent quark mass, $\hat{m}_{q}$, which cannot be extracted from the gap energy, $\epsilon_{k}$,

$$
\begin{aligned}
\epsilon_{k}= & m_{q} s_{k}+k c_{k} \\
& -\frac{2}{3} \int \frac{d \vec{p}}{(2 \pi)^{3}} \hat{V}(|\vec{k}-\vec{p}|)\left[c_{p} c_{k} \hat{k} \cdot \hat{p}+s_{p} s_{k}\right],
\end{aligned}
$$

since $\epsilon_{k}$ diverges for $k \rightarrow 0$. Following previous prescriptions [3-5], we introduce the running dynamic mass, $\hat{m}_{q}^{d y n}(k)$, by $s_{k}=\hat{m}_{q}^{d y n}(k) / \sqrt{\hat{m}_{q}^{d y n}(k)^{2}+k^{2}}$ and obtain $\hat{m}_{q}$ from the slope of the gap angle near zero momentum $\left[\hat{m}_{q} \approx \hat{m}_{q}^{d y n}(0)\right]$. This yields $\hat{m}_{u / d} \approx 80 \mathrm{MeV}$ for $u$, $d$ flavors with $m_{u}=m_{d}=5 \mathrm{MeV}$ and $\hat{m}_{s} \approx 250 \mathrm{MeV}$ for the $s$ quark with $m_{s}=150 \mathrm{MeV}$. Again these values are somewhat lower than those used in phenomenological quark models indicating a more sophisticated vacuum is needed for which we propose the RPA as detailed below.

Using these quasiparticle creation operators we now address excited meson states and first construct the TDA Fock space wave function built on the BCS vacuum. For a meson with quantum numbers $n J^{\pi}$ (radial/node number $n$, total angular momentum $J$, and parity $\pi$ ) the leading $q \bar{q}$ state is given by

$$
\begin{aligned}
&\left|\Psi^{n J^{\pi}}\right\rangle=\sum_{\lambda \mu} \int \frac{d \vec{k}}{(2 \pi)^{3}} \Psi_{\lambda \mu}^{n J^{\pi}}(\vec{k}) B_{\lambda}^{\dagger}(\vec{k}) D_{\mu}^{\dagger}(-\vec{k})|\Omega\rangle, \\
& \Psi_{\lambda \mu}^{n J^{\pi}}(\vec{k})=\sum_{L S m_{L} m_{S}}\left\langle L m_{L} S m_{S} \mid J m_{J}\right\rangle(-1)^{\frac{1}{2}+\mu} \\
& \times\left\langle\frac{1}{2} \lambda \frac{1}{2}-\mu \mid S m_{S}\right\rangle Y_{L}^{m_{L}}(\hat{k}) \psi_{L S}^{n J^{\pi}}(k) .
\end{aligned}
$$

Diagonalizing $H$ in this model space generates the TDA equation of motion (analogous to the Bethe-Salpeter equation)

$$
\left\langle\Psi^{n J^{\pi}}\left|\left[\hat{H}, B_{\alpha}^{\dagger} D_{\beta}^{\dagger}\right]\right| \Omega\right\rangle=\left(E_{n J^{\pi}}-E_{0}\right) \Psi_{\alpha \beta}^{n J^{\pi}} .
$$

Very significantly, the relativistic structure of our effective interaction contains an important spin dependence. This is revealed more clearly in the TDA partial-wave equations for a meson in state $n J^{\pi}$ with mass $M_{n J^{\pi}}$ having quantum numbers $L$ (orbital) and $S$ (total spin)

$$
\left(M_{n J^{\pi}}-2 \epsilon_{k}\right) \psi_{L S}^{n J^{\pi}}(k)=\int_{0}^{\infty} \frac{d p p^{2}}{12 \pi^{2}} K_{L S}^{J^{\pi}}(k, p) \psi_{L S}^{n J^{\pi}}(p),
$$

with kernel $K_{L S}^{J^{\pi}}(k, p)$ structure: (1) pseudoscalar $\left(J^{\pi}=0^{-}\right), \quad 2\left[c_{k} c_{p} \hat{V}_{1}+\left(1+s_{k} s_{p}\right) \hat{V}_{0}\right] ; \quad$ (2) scalar $\left(J^{\pi}=0^{+}\right), \quad 2\left[c_{k} c_{p} \hat{V}_{0}+\left(1+s_{k} s_{p}\right) \hat{V}_{1}\right] ; \quad$ (3) vector $\left(J^{\pi}=1^{-}\right), \quad 2 c_{k} c_{p} \hat{V}_{1}+\left(1+s_{k}\right)\left(1+s_{p}\right) \hat{V}_{0}+(1-$ $\left.s_{p}\right)\left(1-s_{k}\right)\left(\frac{4}{3} \hat{V}_{2}-\frac{1}{3} \hat{V}_{0}\right)$; (4) pseudovector $\left(J^{\pi}=1^{+}\right)$, $c_{k} c_{p}\left(\hat{V}_{0}+\hat{V}_{2}\right)+2\left(1+s_{p} s_{k}\right) \hat{V}_{1}$. Here $\hat{V}_{i}$ is the angular integral over $x=\hat{k} \cdot \hat{q}$ of $\hat{V}(|\vec{k}-\vec{q}|)$ with powers $x^{i}$.

The TDA spectrum is given in Fig. 1 (dotted lines) for the pseudoscalar and vector mesons. Note that in this model isoscalar and isovector states are degenerate and the flavor structure is not predicted. We therefore adopt the standard $\mathrm{SU}_{F}(3)$ flavor mixings for $\eta, \eta^{\prime}, \omega$, and $\phi$. Considering that $\sigma$ is the only, but predetermined, parameter in this model, the spectrum is qualitatively reasonable with the exception of the ground state pion and the higher radial (node number) excited states. From model calculation the spin splitting between the $\rho$ and $\pi$ is about $200 \mathrm{MeV}$, insufficient to describe the roughly $600 \mathrm{MeV}$ observed difference [7]. This shortcoming is due to the inability of the TDA to properly include constraints from chiral symmetry. Regarding the high lying mass states, our predictions are incomplete since a consistent analysis within this model would entail mixing between radial excited one quasiparticle, one quasihole states and more complicated Fock states having two, three, etc. quasiparticle, quasihole (but lower radial) components. Such a calculation is feasible and will be reported in the future.

Finally, we formulate the RPA [8] and generalize the meson creation operator for state $\left|n J^{\pi}\right\rangle=Q_{n J^{\pi}}^{\dagger}\left|\Omega_{\mathrm{RPA}}\right\rangle$,

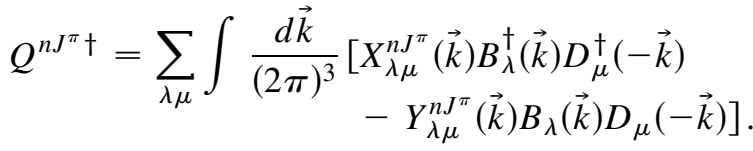

This operator both creates and destroys $q \bar{q}$ pairs from an improved vacuum, satisfying $Q_{n J^{\pi}}\left|\Omega_{\mathrm{RPA}}\right\rangle=0$, which contains quark correlations beyond the BCS. To obtain the RPA equations of motion we make use of the quasiboson approximation [8] in which pairs of operators, $B D$, are treated as boson operators. Using Eq. (9) we replace the BCS vacuum with the RPA and substitute $Q^{\dagger}$ for $B^{\dagger} D^{\dagger}$. For the important pseudoscalar $\left(J^{\pi}=0^{-}\right)$meson channel we obtain the coupled partial-wave RPA equations 


$$
\begin{aligned}
& 2 \epsilon_{k} X^{n}(k)+\frac{1}{3} \int_{0}^{\infty} \frac{d p p^{2}}{(2 \pi)^{2}}\left[X^{n}(p) F(k, p)+Y^{n}(p) G(k, p)\right]=M_{n} X^{n}(k), \\
& 2 \epsilon_{k} Y^{n}(k)+\frac{1}{3} \int_{0}^{\infty} \frac{d p p^{2}}{(2 \pi)^{2}}\left[Y^{n}(p) F(k, p)+X^{n}(p) G(k, p)\right]=-M_{n} Y^{n}(k),
\end{aligned}
$$

where

$$
\begin{aligned}
& F(k, p)=2 c_{p} c_{k} \hat{V}_{1}+2\left(1+s_{p} s_{k}\right) \hat{V}_{0}, \\
& G(k, p)=2 c_{p} c_{k} \hat{V}_{1}-2\left(1-s_{p} s_{k}\right) \hat{V}_{0},
\end{aligned}
$$

with similar expressions for the other meson channels. The RPA spectrum (dashed lines) is summarized in Fig. 1. Notice the improvement with only the pseudoscalar states $(\pi$ and $\eta$ ) shifted downward from the TDA. Related, we now also obtain the correct chiral limit for the pion mass. As expected from chiral arguments concerning the Goldstone boson nature of the pion, the RPA pseudoscalar mass (pure $\mathrm{u} / \mathrm{d}$ flavor) approaches zero for $m_{q} \rightarrow 0$ as illustrated in Fig. 2 (solid curve). Appropriately, the scalar $f_{0}(980)$, which has $L=1, S=1$, mass (dotted curve) converges to a nonzero value $(820 \mathrm{MeV})$ in the same limit (also note, most models significantly overpredict the mass of this controversial meson, our physical prediction is $860 \mathrm{MeV}$ ). Hence the RPA clarifies the major source of mass splitting between the $\pi$ and $\rho$-roughly $200 \mathrm{MeV}$ from spin dependence, as in the TDA, but a much larger amount, about $400 \mathrm{MeV}$, due to chiral symmetry constraints. This finding sharply contrasts with traditional quark models which, while providing good phenomenological mass descriptions, attribute the $\pi$ - $\rho$ mass splitting to one gluon exchange spin dependent potentials [9].

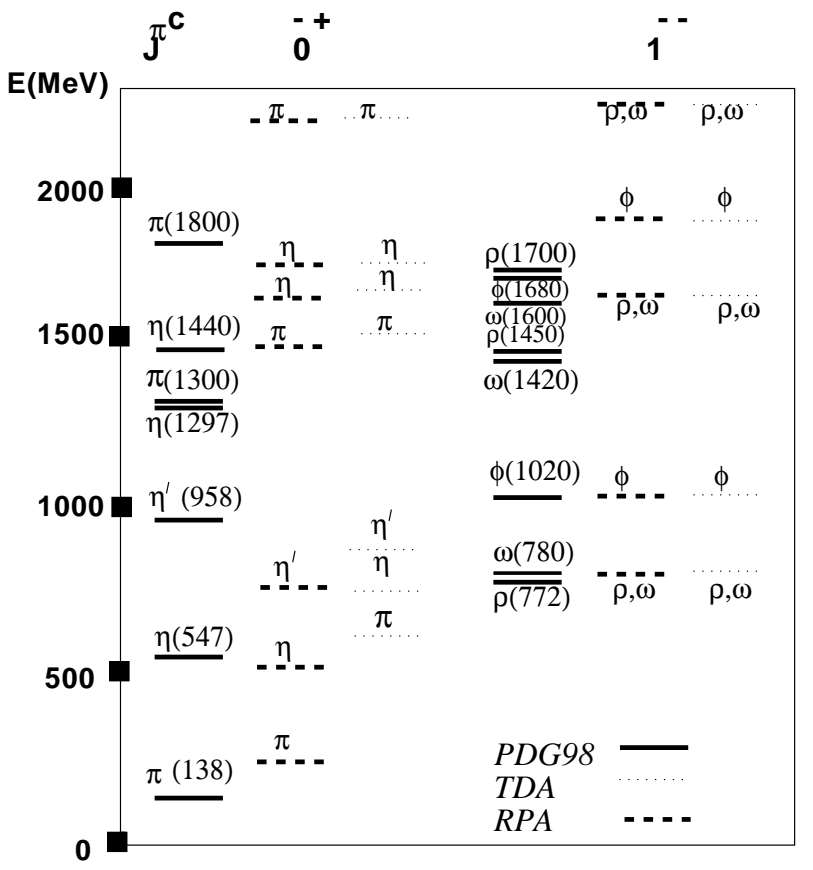

FIG. 1. Pseudoscalar and vector meson spectrum. Data (solid line), RPA (dashed line), and TDA (dotted line).
The RPA also improves the quark condensate and pion decay constant. Performing an expansion of this vacuum in powers of boson operators and keeping only leading corrections exciting up to two mesons from the BCS vacuum, we obtain a much larger condensate. For zero current quark mass, $\langle\bar{q} q\rangle \simeq-(300 \mathrm{MeV})^{3}$, in better agreement with the currently accepted value. Interestingly, this overestimation of ground state correlations seems to be characteristic of RPA, as previously documented in other fields of physics [8]. Finally, in the chiral limit we compute the pion decay constant to this truncated RPA vacuum $f_{\pi}=60 \mathrm{MeV}$ (data $93 \mathrm{MeV}$ ), which is substantially better than the TDA value of $17 \mathrm{MeV}$. Related and also noteworthy, we have numerically verified the generalized Gell-Mann-Oakes-Renner relation, $-2 m_{q}\langle\bar{q} q\rangle=\sum_{n} M_{n}^{2} f_{n}^{2}$, by independently computing terms on both sides of the equation. The excited pseudoscalar states negligibly contribute as their $f_{n}$ are suppressed in the chiral limit.

Summarizing, we have documented the utility of several many-body techniques, especially the RPA, for investigating the QCD structure of hadrons. In conjunction with our previous glueball study we have also further established our effective Hamiltonian and many-body approach as, with just the independent lattice parameter $\sigma$, the semiquantitative features of the vacuum, meson, and glueball spectrums have all been reproduced. Because the approximations are controllable, this framework is

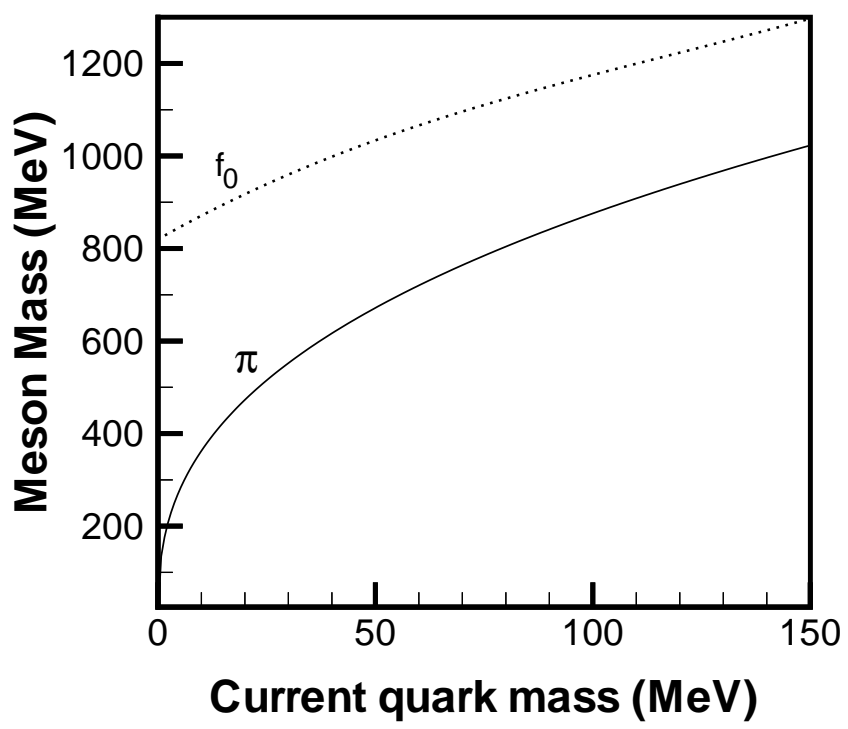

FIG. 2. Chiral symmetry in the RPA. For $m_{q} \rightarrow 0$ the pseudoscalar (solid line) but not scalar (dotted line) meson mass vanishes. 
amenable to systematic improvement and should be appropriate for more challenging hadronic investigations, some in progress, such as baryons, hadron hidden flavor (higher Fock states), and hybrids. The details of the present calculation, as well as several issues concerning renormalization [2] and the $\eta-\eta^{\prime}$ system are deferred to a subsequent, major publication.

The authors recognize useful discussions with J.E. Ribeiro, P. Bicudo, A.P. Szczepaniak, and the NCSU theory group. F. L.-E. acknowledges a SURA-Jefferson Lab fellowship. This work is partially supported by Grants No. DOE DE-FG02-97ER41048 and No. NSF INT-9807009.

[1] A.P. Szczepaniak, E. S. Swanson, C.-R. Ji, and S.R. Cotanch, Phys. Rev. Lett. 76, 2011 (1996); S. R. Cotanch,
A.P. Szczepaniak, E. S. Swanson, and C.-R. Ji, Nucl. Phys. A631, 640 (1998).

[2] D. G. Robertson, A. P. Szczepaniak, E. S. Swanson, C.-R. Ji, and S. R. Cotanch, Phys. Rev. D 59, 074019 (1999).

[3] A. Le Yaouanc, L. Oliver, O. Pene, and J.-C. Raynal, Phys. Rev. D 29, 1233 (1984); A. Le Yaouanc, L. Oliver, S. Ono, O. Pene, and J.-C. Raynal, Phys. Rev. 31, 137 (1985).

[4] P. Bicudo and J.E. Ribeiro, Phys. Rev. D 42, 1611 (1990); 42, 1625 (1990); 42, 1635 (1990), and private communication.

[5] S. L. Adler and A. C. Davis, Nucl. Phys. B244, 469 (1984).

[6] E.S. Swanson and A. P. Szczepaniak, Phys. Rev. D 55, 1578 (1997).

[7] Particle Data Group, Eur. Phys. J. C 3, 1-794 (1998).

[8] P. Ring and P. Schuck, The Nuclear Many-Body Problem (Springer-Verlag, New York, 1980).

[9] S. Godfrey and N. Isgur, Phys. Rev. D 32, 189 (1985). 